\title{
Slip Flow and Heat Transfer of Magnetic Fluids in Micro Porous Media Using a Lattice Boltzmann Method
}

\author{
Xi Chen' ${ }^{1}$, Licong Jin², Xinrong Zhang2* \\ ${ }^{1}$ Department of Mechanics and Engineering Science, College of Engineering, Peking University, Beijing, China \\ ${ }^{2}$ Department of Energy and Resources Engineering, College of Engineering, Peking University, Beijing, China \\ Email: xrzhang@coe.pku.edu.cn
}

Received 26 October 2014; revised 29 November 2014; accepted 21 December 2014

Copyright (C) 2014 by authors and OALib.

This work is licensed under the Creative Commons Attribution International License (CC BY).

http://creativecommons.org/licenses/by/4.0/

(c) (i) Open Access

\begin{abstract}
In this paper, a Lattice Boltzmann method was used to simulate the flow of temperature-sensitive magnetic fluids in a micro porous cavity. According to Navier's linear slip length model, slip boundary conditions were used on all the walls of the micro porous cavity. The effects of the horizontal slip length and the vertical slip length on the flow and heat transfer characteristics were investigated. The results showed that with the increase of the slip length, the velocities and their gradients became smaller, so the convection was harder to occur, and the temperature was more stable. On the walls, the effects of the slip lengths on the Nusselt numbers at the edges and at the centers were different, so the local heat transfer efficiencies were changed accordingly. It was also found that when the horizontal slip length was set to be zero, the flow developed from one vertex to two vortexes along the vertical direction with the increase of vertical slip length. The corresponding critical vertical slip length first increased and then decreased with the Rayleigh number and the magnetic Rayleigh number.
\end{abstract}

\section{Keywords}

Micro Porous Medium, Slip Boundary Condition, Lattice Boltzmann Method, Magnetic Fluid

Subject Areas: Computational Physics, Fluid Mechanics, Numerical Mathematics

\section{Introduction}

Since the 1970s, computational modelings of fluid have flown through micro porous medium developed rapidly [1] [2]. And a Lattice Boltzmann method (LBM) was used to simulate flows in micro porous medium by many researchers [3]-[7]. In their studies, non-slip boundary conditions were presented at the inlet, outlet and other

${ }^{*}$ Corresponding author.

How to cite this paper: Chen, X., Jin, L.C. and Zhang, X.R. (2014) Slip Flow and Heat Transfer of Magnetic Fluids in Micro Porous Media Using a Lattice Boltzmann Method. Open Access Library Journal, 1: e1165.

http://dx.doi.org/10.4236/oalib.1101165 
boundaries; the flows in porous medium were based on macrotransport. Classical Navier-Stokes (N-S) equation and non-slip boundary conditions were used to describe the flow and the heat transfer in micro porous medium.

In fact, macro-scale conditions cannot be directly applied to the micro-scale model, such as the wall boundary of fluid velocity. The experimental results showed that non-slip boundary condition cannot be used in the microand nano-scale models [8]-[10]. Generally, both molecular dynamics (MD) simulation and slip boundary condition are methods to investigate the flow in micro porous media. Plenty of studies focused on the effect of slip boundary on gaseous fluid flow in micro- and nano-porous media [11]-[13]. In above references, the slip boundary conditions were used for gaseous fluid in micro-porous medium. Since the molecular mean free path of the gaseous fluid is much larger than that of the liquid, it is easy for gaseous fluid to be in the slip regime.

The slip flow was not only found in gaseous fluid, but also presented in the liquid motion at the solid interface [14]-[17]. Navier [1] presented the first slip boundary condition, which showed a linear relationship between the slip velocity and the shear rate at the wall. Craig et al. [8] experimentally measured the slip length and found it to be a function of the liquid viscosity and the shear rate. Thompson and Troian [14] found that the slip velocity was related to the slip length, the shear rate at the wall and a critical shear rate at which the slip length diverged. Their model was widely used in current studies in micro and nano flow problems [17] [18].

The natural convection of magnetic fluids in porous media was studied, using a Lattice Boltzmann simulation [19] [20]. However, whether the scale of porous media affects the flow and heat transfer mechanism is still unknown. It may have widely applications in the industry. For instance, magnetic fluids may be used in the cooling of micro-electromechanical systems. Thus, the investigation of magnetic fluids in micro porous media is required. In the present study, a Lattice Boltzmann method is used to simulate the flow of temperature-sensitive magnetic fluids in a micro porous cavity, containing the slip boundaries on all the walls. The effects of the horizontal slip length and the vertical slip length on the flow and heat transfer characteristics were investigated.

\section{Physical Model and Numerical Method}

\subsection{Physical Model}

We present the physical model in Figure 1 which is a square micro-porous cavity. The square micro-porous cavity is of length $L(L=5 \mu \mathrm{m})$ filled with magnetic fluid, heated from the bottom wall and cooled from the upper wall with constant temperature $T_{\text {heat }}$ and $T_{\text {cool }}$. Both of the two vertical side walls are thermally insulated. The initial temperature $T_{0}$ (reference temperature) is set as $298.15 \mathrm{~K}$ in whole calculation domain and it is equal to $T_{\text {cooll }}$. We assume the porosity $\varepsilon$ of micro-porous medium to be 0.4 with permeability $K=2.3 \times 10^{-8}$. The magnetic fluid in porous medium is magnetized in a uniform magnetic intensity $H_{0}$ along the y axis, its direction opposites to gravitational acceleration $(g)$. In order to investigate the effect of slip velocity, the magnetic Rayleigh number $\left(\operatorname{Ram}=\mu_{0} H_{0} M_{s} L^{2} / k \eta\right)$ is set to be constant $\operatorname{Ram}=1.0 \times 10^{4}$.

$$
T_{2}=T_{\text {cool }}
$$

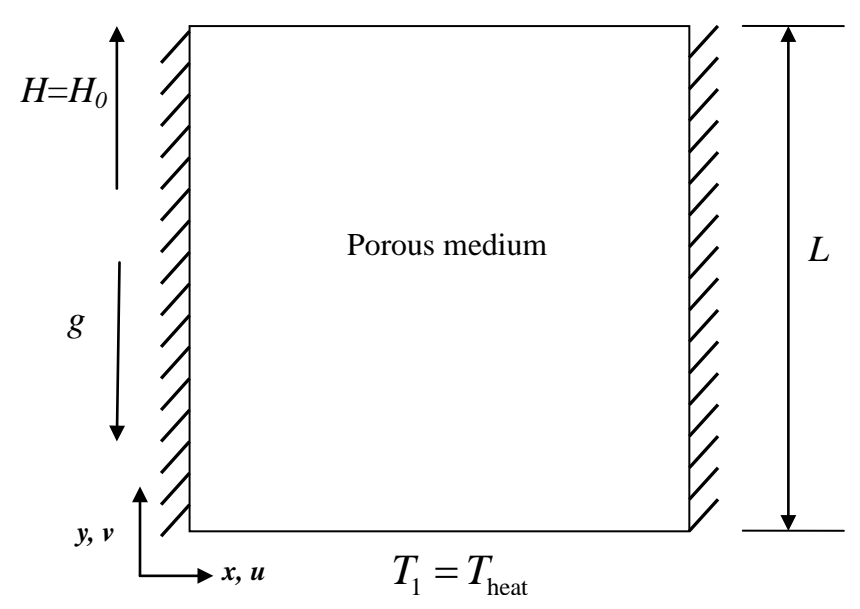

Figure 1. Physical model of micro porous cavity. 


\subsection{The Lattice Boltzmann Model for Magnetic Fluid in Porous Medium}

In the simulation, the cavity modeled is assumed to be filled with isotropic, homogeneous and non-deformable porous medium. With these assumptions, the dimensionless governing equations: continuity equation, momentum equation and energy equation containing the combined effect of magnetic field and porous medium are described as Equations (1)-(3). Besides, an elliptic equation for magnetic potential, which is a function related closely to magnetic intensity $H_{0}$ and the magnetization $M$ are reduced to Equation (4) [19].

$$
\begin{gathered}
\nabla \cdot \boldsymbol{u}=0 \\
\partial_{t}(\rho \boldsymbol{u})+\nabla \cdot\left(\frac{1}{\varepsilon} \rho \boldsymbol{u} \boldsymbol{u}\right)=-\nabla(\varepsilon p)+\eta \nabla^{2} \boldsymbol{u}+\boldsymbol{F} \\
\sigma \partial_{t} T+\boldsymbol{u} \cdot \nabla T=\frac{\lambda\left(T_{c}-T_{0}\right)}{\left[\rho C_{p}\left(T_{c}-T_{0}\right)+\mu_{0} \chi_{0} H^{2}\right]} \nabla^{2} T+S \\
\frac{1}{\gamma} \partial_{t} \phi+\boldsymbol{u}_{T} \cdot \nabla \phi=\left[1+\frac{\chi_{0}\left(T-T_{0}\right)}{T_{c}-T_{0}}\right] \nabla^{2} \phi
\end{gathered}
$$

where $\boldsymbol{F}$ is the total force, including magnetic force, efficient gravity, viscous loss term (Darcy) and geometric loss term in porous medium, $S$ is the energy source, $\sigma$ represents the ratio between the heat capacities of the porous medium and that of magnetic fluid phases. They are given as following:

$$
\begin{gathered}
\boldsymbol{F}=\frac{\chi_{0} \mu_{0} H^{2}}{2\left(T_{c}-T_{0}\right)} \nabla T-\varepsilon \rho g \beta\left(T-T_{0}\right)-\frac{\varepsilon \eta}{K} \boldsymbol{u}-\frac{\varepsilon F_{\varepsilon}}{\sqrt{K}}|\boldsymbol{u}| \mathbf{u} \\
F_{\varepsilon}=\frac{1.75}{\sqrt{150(1-\varepsilon)^{2}}} \\
S=-\frac{\chi_{0} \mu_{0} H T \frac{\mathrm{d} H}{\mathrm{~d} t}}{\rho C_{p}\left(T_{c}-T\right)+\chi_{0} \mu_{0} H^{2}} \\
\sigma=\varepsilon+(1-\varepsilon) \frac{\rho^{\prime} C_{p}^{\prime}}{\rho C_{p}}
\end{gathered}
$$

It is noted that Equation (4) is the magnetic potential equation, where $\phi$ is the scalar potential, the relationship between $\phi$ and $H$ is $H=\nabla \phi . \gamma$ is the adjustable preconditioning parameter in analysis. $\boldsymbol{u}_{T}$ is defined as effective velocity, and it is given as:

$$
\boldsymbol{u}_{T}=\frac{\chi_{0} \nabla T}{T_{c}-T_{0}}
$$

In all the equations, $\rho, \lambda, C_{p}, \eta, \chi_{0}, \mu_{0}, \beta$ are respectively the fluid density, thermal conductivity, specific heat, viscosity, magnetization rate, permeability of vacuum and expansion coefficient. $\rho^{\prime}, C_{p}^{\prime}, \varepsilon$ are the density, thermal conductivity and porosity for the porous medium.

Following the lattice Boltzmann method of D2Q9 model, the description of the velocity, temperature and magnetic potential, which is the dispersion for Equations (1)-(4), are written by the following equations:

$$
\begin{gathered}
f_{i}\left(r+\xi_{i} \delta_{t}, t+\delta_{t}\right)-f_{i}(r, t)=\frac{f_{i}(r, t)-f_{i}^{e q}(r, t)}{\tau_{f}}+w_{i} \frac{\left(\tau_{f}-0.5\right) \delta_{t}}{\tau_{f} c_{s}^{2}} \cdot \boldsymbol{F} \cdot\left(\xi_{i}-\boldsymbol{u}\right) \\
g_{i}\left(r+\xi_{i} \delta_{t}, t+\delta_{t}\right)-g_{i}(r, t)=-\frac{g_{i}(r, t)-g_{i}^{e q}(r, t)}{\tau_{g}}+w_{i} S \delta_{t} \\
h_{i}\left(r+\xi_{i} \delta_{t}, t+\delta_{t}\right)-h_{i}(r, t)=-\frac{h_{i}(r, t)-h_{i}^{e q}(r, t)}{\tau_{h}}
\end{gathered}
$$


where the equilibrium distribution functions for the above equations are:

$$
\begin{gathered}
f_{i}^{e q}(r, t)=w_{i} \rho\left\{1+\frac{\xi_{i} \cdot \boldsymbol{u}}{c_{s}^{2}}+\frac{1}{2 c_{s}^{2}}\left(\frac{\left(\xi_{i} \cdot \boldsymbol{u}\right)^{2}}{c_{s}^{2}}-\boldsymbol{u}^{2}\right)\right\} \\
g_{i}^{e q}(r, t)=w_{i} \frac{D T}{2}\left\{\frac{\xi_{i}^{2}}{D c_{s}^{2}}+\frac{\xi_{i} \cdot \boldsymbol{u}}{D c_{s}^{2}}\left(\frac{\xi_{i}^{2}}{c_{s}^{2}}-2\right)+\frac{1}{2 c_{s}^{2}}\left(\frac{\left(\xi_{i} \cdot \boldsymbol{u}\right)^{2}}{c_{s}^{2}}-\boldsymbol{u}^{2}\right)\right\} \\
h_{i}^{e q}(r, t)=w_{i} \phi\left\{1+\frac{\xi_{i} \cdot \gamma \boldsymbol{u}_{T}}{c_{s}^{2}}+\frac{1}{2 c_{s}^{2}}\left(\frac{\left(\xi_{i} \cdot \gamma \boldsymbol{u}_{T}\right)^{2}}{c_{s}^{2}}-\left(\gamma \boldsymbol{u}_{T}\right)^{2}\right)\right\}
\end{gathered}
$$

The dimensionless relaxation parameters in the Equations (10)-(12) are determined by the effective vescosity, thermal conductivity and magnetization rate:

$$
\begin{gathered}
\tau_{f}=\frac{\eta}{\rho c_{s}^{2} \delta_{t}}+0.5 \\
\tau_{g}=\frac{1}{\sigma} \cdot \frac{D}{D+2} \cdot \frac{\lambda\left(T_{c}-T\right)}{\left[\rho C_{p}\left(T_{c}-T\right)+\chi_{0} \mu_{0} H^{2}\right] c_{s}^{2} \delta_{t}}+0.5 \\
\tau_{h}=\frac{\gamma}{c_{s}^{2} \delta_{t}}\left[1+\frac{\chi_{0}\left(T-T_{0}\right)}{T_{c}-T}\right]+0.5
\end{gathered}
$$

The volume-averaged density, velocity, temperature and magnetic potential are defined as follows:

$$
\begin{gathered}
\rho=\sum_{i} f_{i} \\
\boldsymbol{u}=\frac{\boldsymbol{u}^{\prime}}{c_{0}+\sqrt{c_{0}^{2}+c_{1}\left|\boldsymbol{u}^{\prime}\right|}} \\
T=\sum_{i} g_{i} \\
\phi=\sum_{i} h_{i}
\end{gathered}
$$

where $c_{0}, c_{1}$ and $\boldsymbol{u}^{\prime}$ can be described as:

$$
\begin{gathered}
c_{0}=\frac{1}{2}\left(1+\frac{\delta_{t}}{2} \cdot \frac{\varepsilon \eta}{K}\right) \\
c_{1}=\frac{\delta_{t}}{2} \cdot \frac{\varepsilon F_{\varepsilon}}{K} \\
\rho \boldsymbol{u}^{\prime}=\sum_{i} f_{i} \xi_{i}+0.5 \delta_{t} \mathbf{F}
\end{gathered}
$$

In this paper, the non-dimensionless vertical velocity, horizontal velocity and temperature are defined as:

$$
v^{*}=\frac{v_{l}}{u_{0}}, \quad u^{*}=\frac{u_{l}}{u_{0}}, \quad T^{*}=\frac{T_{l}-T_{0}}{\Delta T}
$$

where $v_{l}, u_{l}$ and $T_{l}$ are local vertical velocity, horizontal velocity and temperature. And $u_{0}, T_{0}$ are respectively reference velocity and reference temperature. $u_{0}$ is given from follow equation:

$$
u_{0}=\sqrt{g \beta(\Delta T) L}
$$

\subsection{Slip Boundary Conditions}

In micro and nano scales, the fluid properties and interfacial roughness must be considered, so the non-slip 
boundary condition is not suitable for the small scale, which can be confirmed by the experimental results [8][10].

The slip boundary condition described by Thompson and Troian [14] can be written as

$$
\hat{u}_{x=0, L}=L_{y}^{0} \dot{\gamma}_{w}\left(1-\frac{\dot{\gamma}_{w}}{\dot{\gamma}_{c}}\right)^{-1 / 2}, \hat{u}_{y=0, L}=L_{x}^{0} \dot{\gamma}_{w}\left(1-\frac{\dot{\gamma}_{w}}{\dot{\gamma}_{c}}\right)^{-1 / 2}
$$

where $L_{x}^{0}$ and $L_{y}^{0}$ are the constant slip lengths along x-axis and y-axis, respectively, $\dot{\gamma}_{w}$ the shear rate at the wall, and $\dot{\gamma}_{c}$ the critical shear rate at which the slip length diverges. As shown by Yang [17], the assumption of $\dot{\gamma}_{w} / \dot{\gamma}_{c} \ll 1$ is reasonable in the slip-slow situation. So the slip velocities can be written as:

$$
\hat{u}_{x=0, L}=L_{y}^{0} \frac{\partial u_{y}}{\partial x}, \quad \hat{u}_{y=0, L}=L_{x}^{0} \frac{\partial u_{x}}{\partial y}
$$

The rheology can be expressed as:

$$
\hat{\tau}_{x}=-\frac{y}{2} \frac{\mathrm{d} p}{\mathrm{~d} x}=\eta\left(-\frac{\mathrm{d} \hat{u}_{x}}{\mathrm{~d} y}\right), \quad \hat{\tau}_{y}=-\frac{x}{2} \frac{\mathrm{d} p}{\mathrm{~d} y}=\eta\left(-\frac{\mathrm{d} \hat{u}_{y}}{\mathrm{~d} x}\right)
$$

where $\hat{\tau}_{x}$ and $\hat{\tau}_{y}$ are the shear stresses, $p$ the pressure, $\eta$ the viscosity. Combining Equations (27) and (28), the velocity profiles can be given as:

$$
\hat{u}_{x}=\left(-\frac{1}{2 \eta} \frac{\mathrm{d} p}{\mathrm{~d} x}\right)\left(L L_{y}^{0}+\left(\frac{1}{4} L^{2}-x^{2}\right)\right), \quad \hat{u}_{y}=\left(-\frac{1}{2 \eta} \frac{\mathrm{d} p}{\mathrm{~d} y}\right)\left(L L_{x}^{0}+\left(\frac{1}{4} L^{2}-y^{2}\right)\right)
$$

With the maximum velocity at $x=0$ and $y=0$,

$$
\left.\hat{u}_{x}\right|_{\max }=\left(-\frac{1}{2 \eta} \frac{\mathrm{d} p}{\mathrm{~d} x}\right)\left(L L_{y}^{0}+\frac{1}{4} L^{2}\right),\left.\quad \hat{u}_{y}\right|_{\max }=\left(-\frac{1}{2 \eta} \frac{\mathrm{d} p}{\mathrm{~d} y}\right)\left(L L_{x}^{0}+\frac{1}{4} L^{2}\right)
$$

Non-dimensional velocity profile can be express as:

$$
\begin{aligned}
& u_{x}=\frac{\hat{u}_{x}}{\left.\hat{u}_{x}\right|_{\max }}=\frac{L L_{y}^{0}+\left(\frac{1}{4} L^{2}-x^{2}\right)}{L L_{y}^{0}+\frac{1}{4} L^{2}}=1-\left(\frac{\psi_{x} x}{L / 2}\right)^{2} \\
& u_{y}=\frac{\hat{u}_{y}}{\left.\hat{u}_{y}\right|_{\max }}=\frac{L L_{x}^{0}+\left(\frac{1}{4} L^{2}-y^{2}\right)}{L L_{x}^{0}+\frac{1}{4} L^{2}}=1-\left(\frac{\psi_{y} y}{L / 2}\right)^{2}
\end{aligned}
$$

where $\psi_{x}=\left(\frac{1}{4 L_{y}^{0} / L+1}\right)^{1 / 2}$ and $\psi_{y}=\left(\frac{1}{4 L_{x}^{0} / L+1}\right)^{1 / 2}$. Then non-dimensional slip velocity is a function of the slip length. The slip boundary conditions can be given as following:

$$
u_{x=0, L}=0, \quad u_{y=0, L}=0
$$

Equation (31) is the non-dimensionless velocity employing the slip velocity boundary condition. It can be dispersed into the Lattice Boltzmann equations (Equations (23) and (24)) in Section 2.2. Then we can solve the LB equations to analyze the effect of slip length in micro porous medicum.

In this paper, two physical dimensionless parameters are defined to describe the slip lengths. The effect of the horizontal slip length and the vertical slip length can be described by two non-dimensionless parameters $a$ and $b$ respectively.

$$
a=\frac{L_{x}^{0}}{L / \text { lattices }}, \quad b=\frac{L_{y}^{0}}{L / \text { lattices }}
$$

where $L$ is the length of square porous cavity, lattices is the lattice number on the slip boundary, $L_{x}$ and $L_{y}$ are 
the slip lengths along $\mathrm{x}$-axis and $\mathrm{y}$-axis. The whole domain is meshed to be $50 \times 50$ lattices. The corresponding relationship between slip length and non-dimensionless length is given as following:

$$
\begin{aligned}
& L_{x}^{0}=1.0 \times 10^{-7} \mathrm{~m}, \quad a=0.1, \\
& L_{x}^{0}=1.0 \times 10^{-8} \mathrm{~m}, \quad a=0.01, \\
& L_{x}^{0}=1.0 \times 10^{-9} \mathrm{~m}, \quad a=0.001, \\
& L_{y}^{0}=1.0 \times 10^{-7} \mathrm{~m}, \quad b=0.1, \\
& L_{y}^{0}=1.0 \times 10^{-8} \mathrm{~m}, \quad b=0.01, \\
& L_{y}^{0}=1.0 \times 10^{-9} \mathrm{~m}, \quad b=0.001 .
\end{aligned}
$$

The Rayleigh number $(R a)$, the magnetic Rayleigh number $(R a m)$ and the Darcy number $(D a)$ are defined as:

$$
R a=\frac{\rho g \beta \Delta T L^{3}}{k \eta}, \quad R a m=\frac{\mu_{0} H_{0} M_{s} L^{2}}{k \eta}, \quad D a=\frac{K}{L^{2}}
$$

The local Nusselt number on heated wall and cooled wall are written as $N u_{h}$ and $N u_{c}$ for convenience. They are defined as:

$$
\begin{aligned}
& N u_{h}=\frac{h l}{\lambda}=\frac{L}{\Delta T} \times\left.\frac{\partial T}{\partial y}\right|_{\partial y \rightarrow 0, y=0}=\frac{(\Delta T)_{h}}{\Delta T} \cdot \frac{L}{(\Delta y)_{h}} \\
& N u_{c}=\frac{h l}{\lambda}=\frac{L}{\Delta T} \times\left.\frac{\partial T}{\partial y}\right|_{\partial y \rightarrow 0, y=1}=\frac{(\Delta T)_{c}}{\Delta T} \cdot \frac{L}{(\Delta y)_{c}}
\end{aligned}
$$

where $(\Delta T)_{h},(\Delta T)_{c}$ are temperature differences defined as:

$$
\begin{aligned}
& (\Delta T)_{h}=T_{(\Delta y)_{h}}-T_{h} \\
& (\Delta T)_{c}=T_{(\Delta y)_{c}}-T_{c}
\end{aligned}
$$

In above equations, $\Delta T$ is the difference between the heated and cooled wall $\left(\Delta T=T_{h}-T_{c}\right) \cdot(\Delta y)_{h},(\Delta y)_{c}$ are the infinite small distance from the heated wall and cooled wall at $y$ axis. $T_{(\Delta y)_{h}}, T_{(\Delta y)_{c}}$ are represented as temperature on the plane of $(\Delta y)_{h}$ and $T_{(\Delta y)_{c}}$.

The average Nusselt number on heated wall $\left(\overline{N u_{h}}\right)$, average Nusselt number on cooled wall $\left(\overline{N u_{c}}\right)$ and average Nussellt number $(\overline{\mathrm{Nu}})$ are respectively defined as:

$$
\begin{aligned}
& \overline{N u_{h}}=\frac{\int_{0}^{L} N u_{h} \mathrm{~d} x}{L} \\
& \overline{N u_{c}}=\frac{\int_{0}^{L} N u_{c} \mathrm{~d} x}{L} \\
& \overline{N u}=\frac{\overline{N u_{h}}+\overline{N u_{c}}}{2}
\end{aligned}
$$

\section{Results and Discussion}

In this section, the magnetic Rayleigh number, porosity of porous cavity and Darcy number are given constant value, as $\operatorname{Ram}=1.0 \times 10^{-8}, \varepsilon=0.4$ and $D a=10^{-3}$. The isotherms, streamlines and the Nusselt number are presented to investigate the effects of the horizontal slip length and the vertical slip length.

\subsection{Effect of the Horizontal Slip Length}

In this subsection, in order to study the effect of the horizontal slip length on the flow and the heat transfer characteristics, the vertical slip length $b$ is set to be zero. 
Figure 2 shows the distribution of streamlines and isotherms with the variation of the horizontal slip length. It can be seen that when the horizontal slip length becomes larger, the global flow will be more stable. It means the convection will be harder to occur at larger horizontal slip length. The physical reason is, the velocities of the flow will decrease with the horizontal slip length, then the convection will be restrained. We can see in flow fields that when horizontal slip length becomes larger, the cores of the vortexes will be close to the vertical walls. It is because the horizontal velocity of the flow becomes smaller but the vertical velocity is relatively stable. However, flow field always has two vortexes, bifurcation of the flow does not occur. The physical reason is the variation of flow fields is restricted by the boundaries.

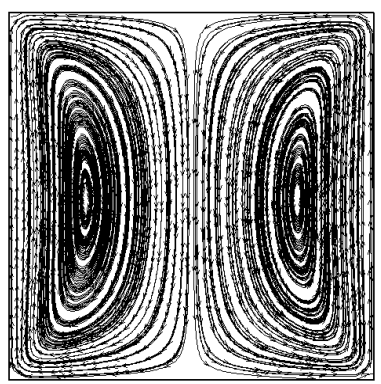

(a)

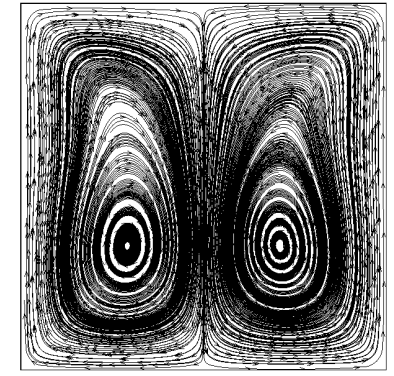

(c)

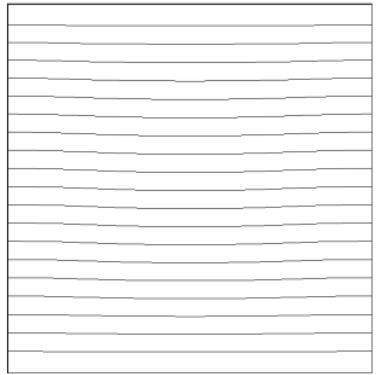

(a)

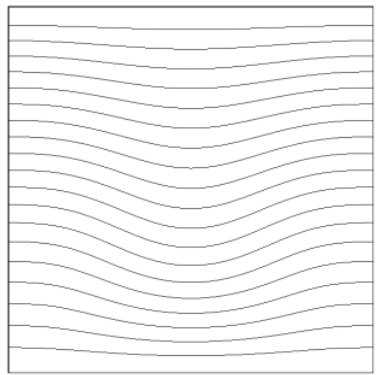

(c)

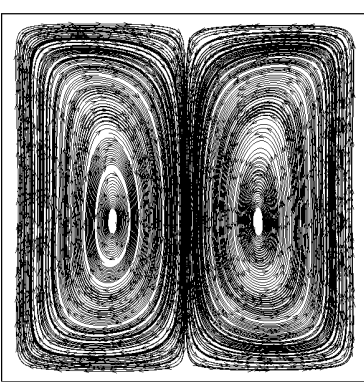

(b)

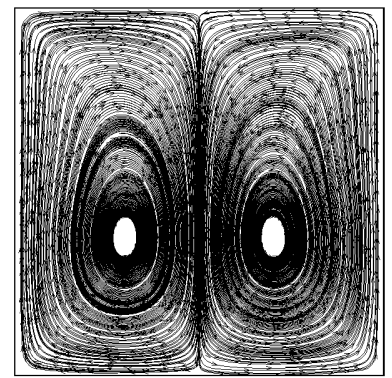

(d)

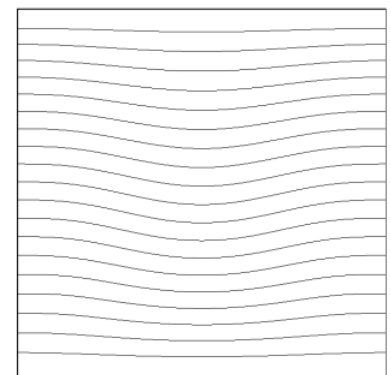

(b)

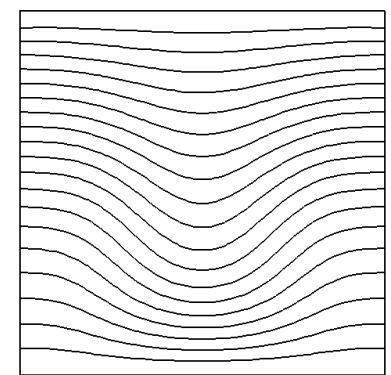

(d)

Figure 2. The streamlines (top) and isotherms (bottom) at different horizontal slip lengths (a) $\mathrm{a}=0.1$; (b) $\mathrm{a}=0.01$; (c) $\mathrm{a}=0.001$; (d) $\mathrm{a}=0$. 
The variation of the whole velocity field is shown in Figure 3. When the horizontal slip length becomes larger, the horizontal velocity of the flow will decrease obviously, and so will the horizontal velocity gradient, the convection will be harder to occur. Moreover, the vertical velocity is affected by the whole flow field. It decreases with the horizontal slip length, but the variation of the vertical velocity gradient is not as large as that of the horizontal velocity gradient. That is the physical explains of the distributions of streamlines and isotherms in details.

Figure 4 and Figure 5 show the variations of Nusselt numbers on the cooled wall $\left(N u_{c}\right)$, heated wall $\left(N u_{h}\right)$ and the average Nusselt number. It can be seen that Nusselt numbers on the cooled wall decrease with $a$, but Nusselt number in the middle increases with $a$ (Figure 4(a)). The physical reason is when the horizontal slip length becomes larger, the velocity gradients and the temperature gradients on the cooled wall will decrease, the local convection will be harder to occur, the heat transfer efficiency will be lower, so the Nusselt numbers will be smaller; but the velocity gradient and the temperature gradient in the middle of the region will increase so the Nusselt number will be larger. On the other hand, on the heated wall, magnetic field force is small, it is weaker than force of gravity, the velocity gradients and the temperature gradients on the wall increase with a, the local convection will be easier to occur, the heat transfer efficiency will raise, so the Nusselt number will be larger; but the velocity gradient and the temperature gradient in the middle of the region decrease with a, the local convection will be harder to occur, heat transfer efficiency will descend, so the Nusselt number will be smaller. It can be seen in Figure 5 that when the horizontal slip length becomes larger, the average Nusselt number of the whole cavity will be smaller. It means the whole convection will be harder to occur, and the heart transfer efficiency will descend with the increase of the horizontal slip length.
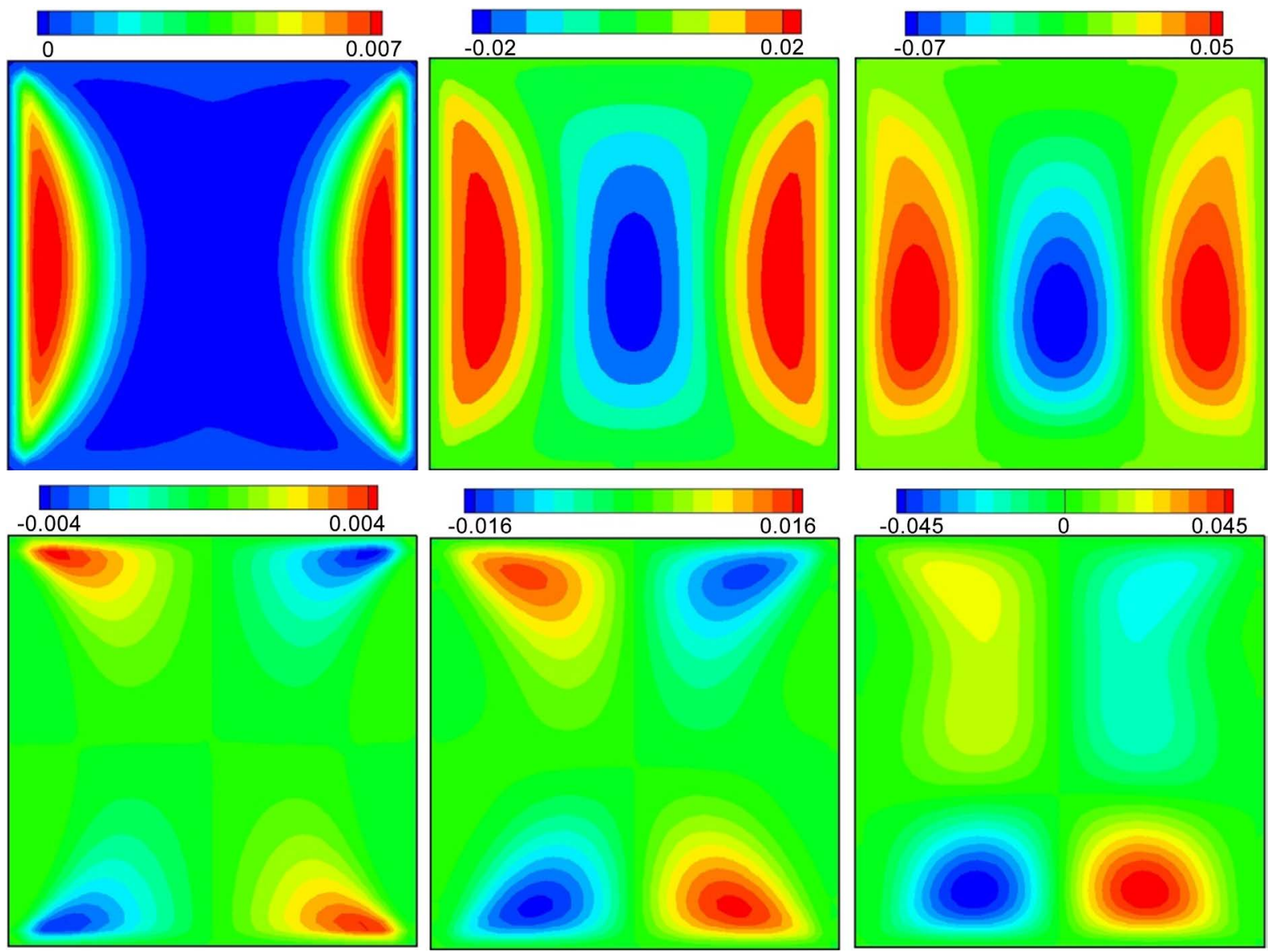

(a) $\mathrm{a}=0.1$

(b) $\mathrm{a}=0.01$

(c) $\mathrm{a}=0.001$

Figure 3. The contours of dimensionless vertical velocity (top) and dimensionless horizontal velocity (bottom) at different horizontal slip lengths. 


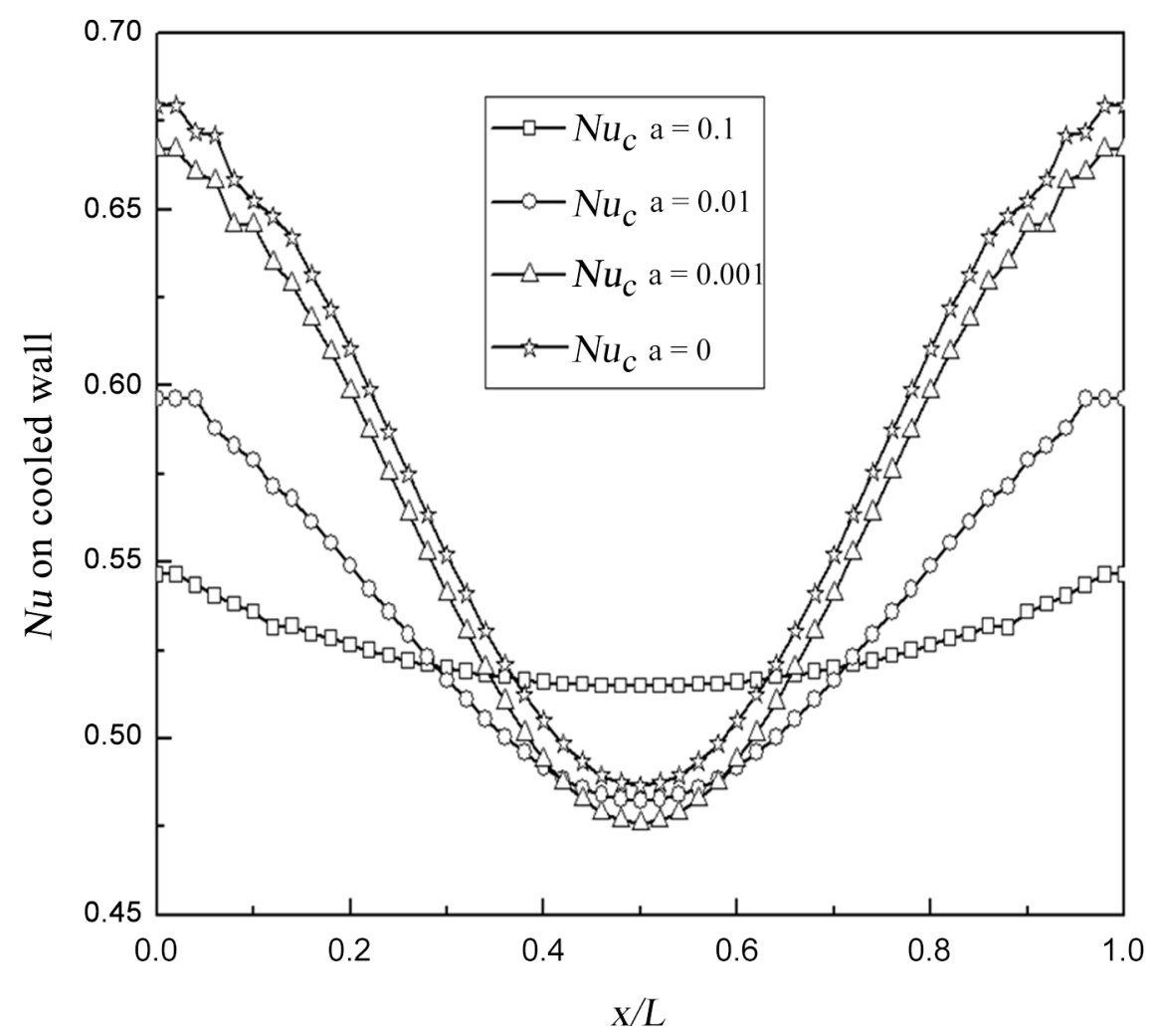

(a)

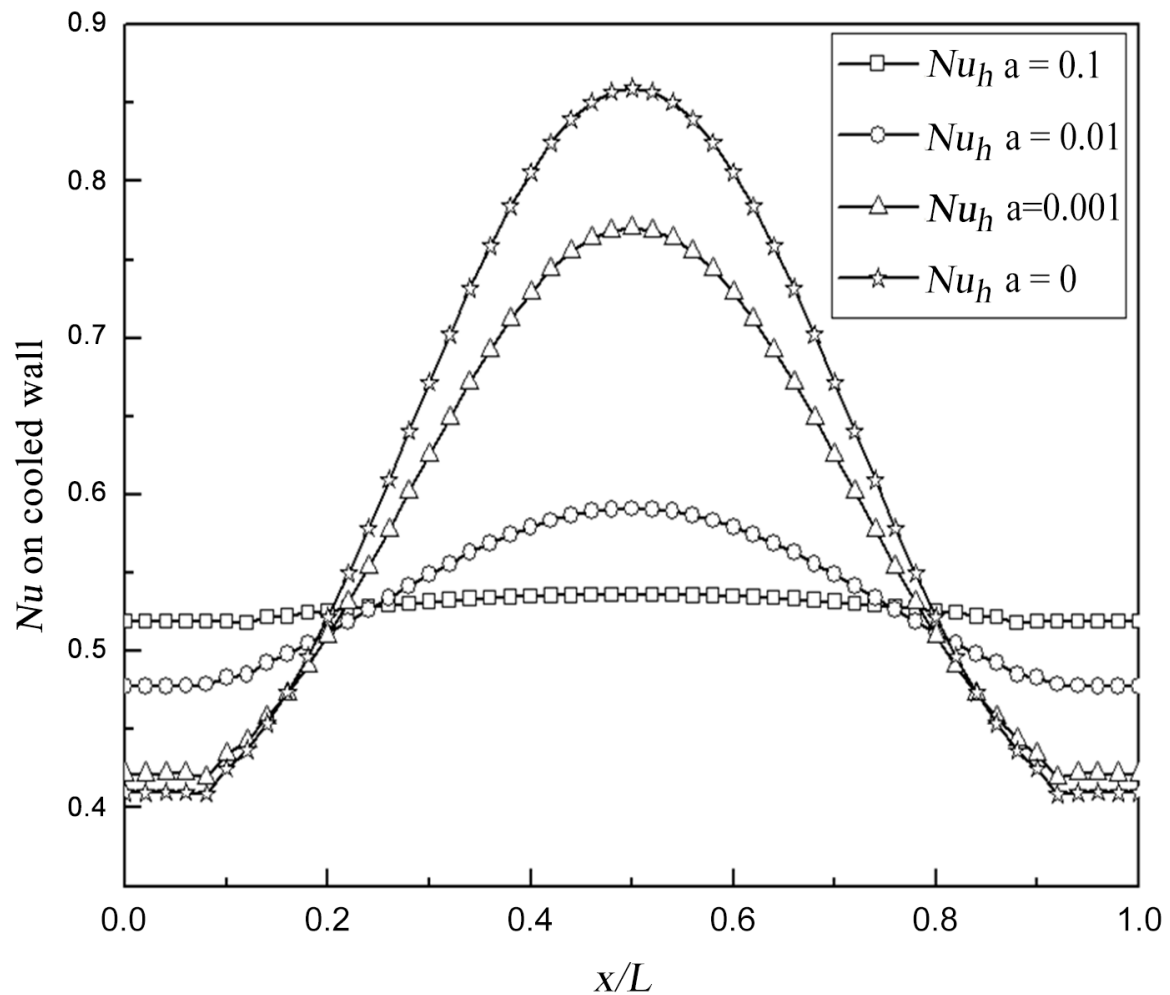

(b)

Figure 4. (a) The local Nusselt number on cooled upper wall; (b) The local Nusselt number on heated bottom wall changing with horizontal slip length. 


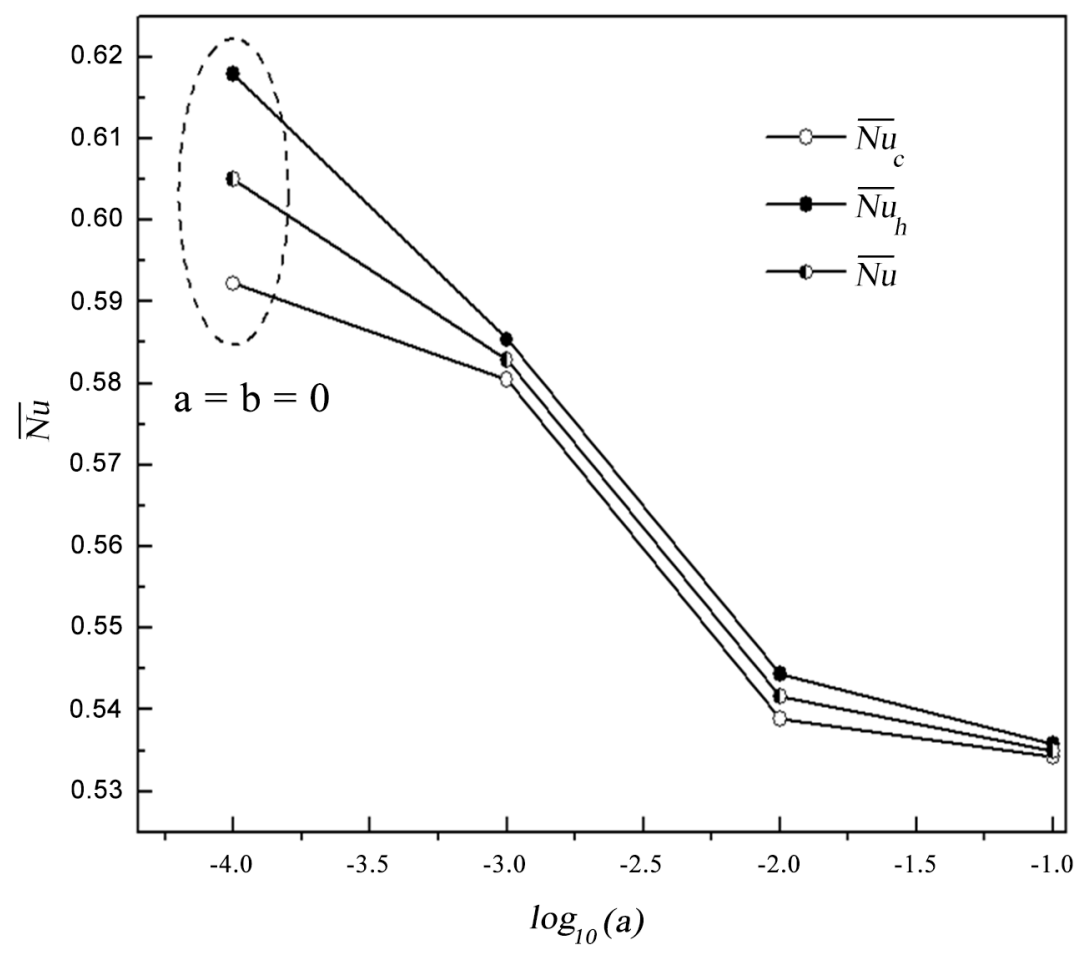

Figure 5. The average Nusselt number changing with horizontal slip length.

\subsection{Effect of the Vertical Slip Length}

In this subsection, the horizontal slip length is set to be zero to study the effects of the vertical slip length on the flow and the heat transfer characteristics.

Figure 6 shows the distribution of streamlines and isotherms with the variation of vertical slip length. When the vertical slip length becomes larger, the convection will be harder to occur. Different from the effect of horizontal slip length, when $b$ becomes larger, two vortexes develop to four vortexes. It is because that the heat driving force will be smaller when the system becomes more stable, then the vertical velocity will be smaller and not enough to drive the convection in the whole cavity (Figure 7(a)). With the increase of $b$, the horizontal and vertical velocity and the velocity gradients decrease, the convection will be restrained. The variation of the vertical velocity gradient is smaller than that of the horizontal velocity gradient.

It can be found in Figure 8 that on the cooled wall, the Nusselt number $\left(N u_{c}\right)$ decreases with $b$ near side walls, but at the center of the wall, the Nusselt number increases with $b$. On the heated wall, the Nusselt number $\left(N u_{h}\right)$ increases with $b$ near side walls, but at the center, the Nusselt number decreases with $b$. The average Nusselt number decreases with $b$, so the whole convection will be restrained, the heat transfer efficiency will descend. Comparing Figure 5 and Figure 9, we can find that the effect of the vertical slip length is more obvious than that of the horizontal slip length.

In Figure 6, we find the flow develops from two vortexes to four vortexes with the increase of the vertical slip length, so we define a critical slip length to describe the critical conditions that the flow regime changes. In order to investigate the critical condition that the convection of the whole cavity occurs, we calculate and summarize the critical slip lengths under the different value of the Rayleigh number and the magnetic Rayleigh number. It can be seen in Figure 10 that the critical vertical slip length increases and then decreases with Rayleigh number and magnetic Rayleigh number. When Rayleigh number and magnetic Rayleigh number are small, the increase of Rayleigh number and magnetic Rayleigh number will induce the convection to occur, the vertical slip length will hinder the convection to occur. When the slip length is large, it is need higher magnetic field and difference in temperature to drive the convection to occur. Therefore, when the Rayleigh number and the magnetic Rayleigh number raise, the critical vertical slip length will be larger. When the Rayleigh number and the magnetic Rayleigh number are large, the magnetic force on the magnetic fluid will raise because of the raise of difference in temperature and magnetic field. The direction of magnetic force is opposite to the gravity, the con- 


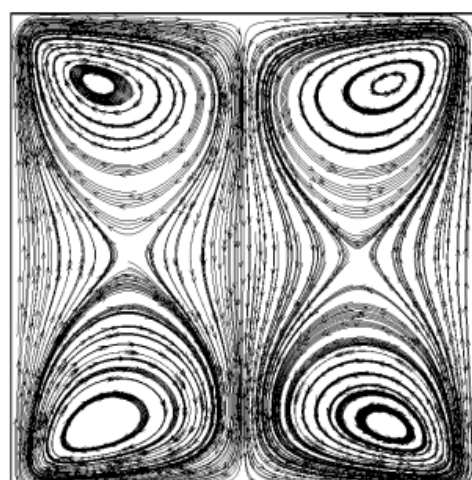

(a)

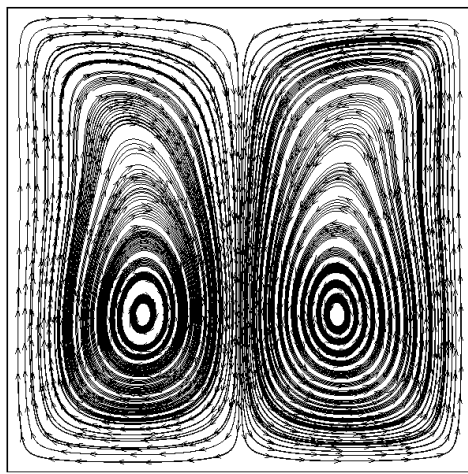

(c)

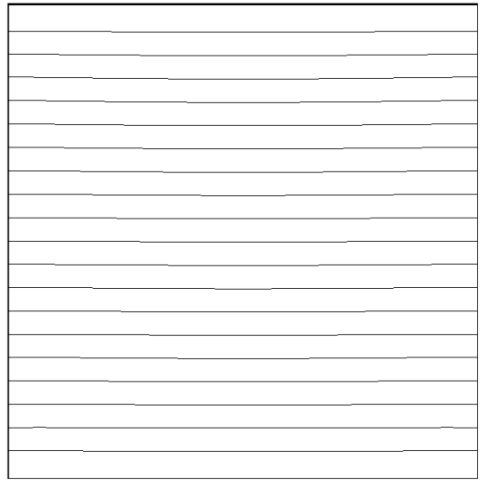

(a)

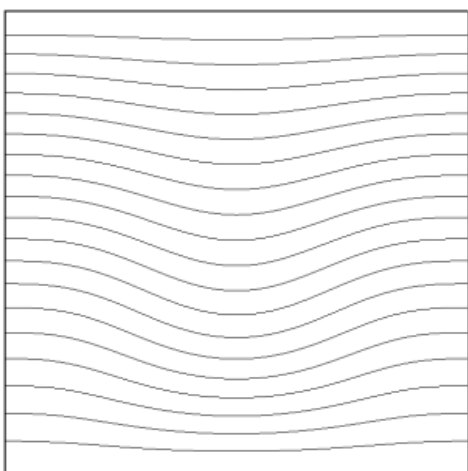

(c)

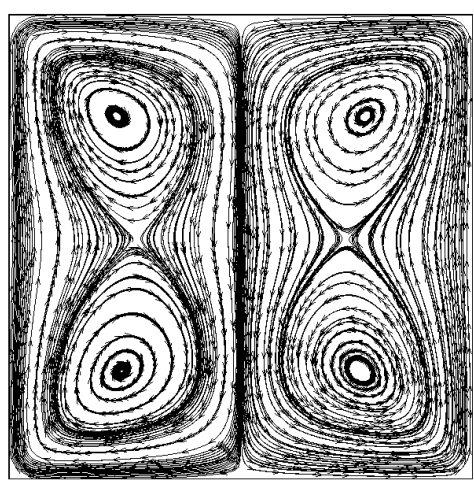

(b)

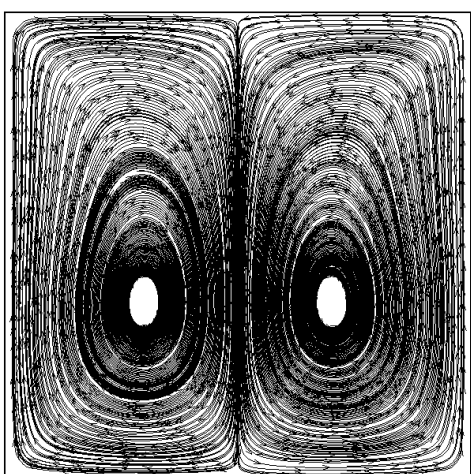

(d)

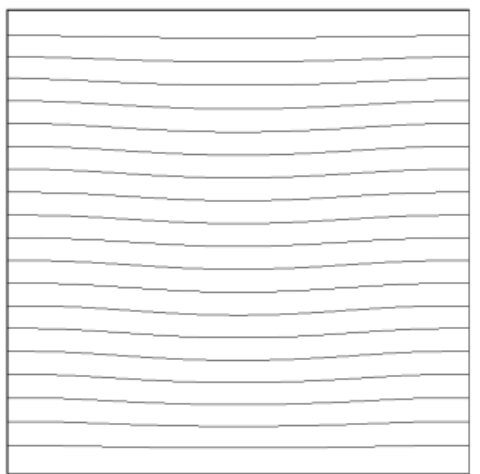

(b)

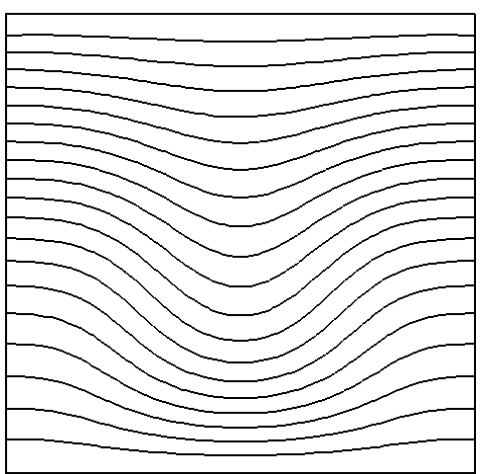

(d)

Figure 6. The streamlines (top) and isotherms (bottom) at different vertical slip lengths (a) $b=0.1 ;$ (b) $b=0.01 ;$ (c) $b=0.001 ;$ (d) $b=0$. 


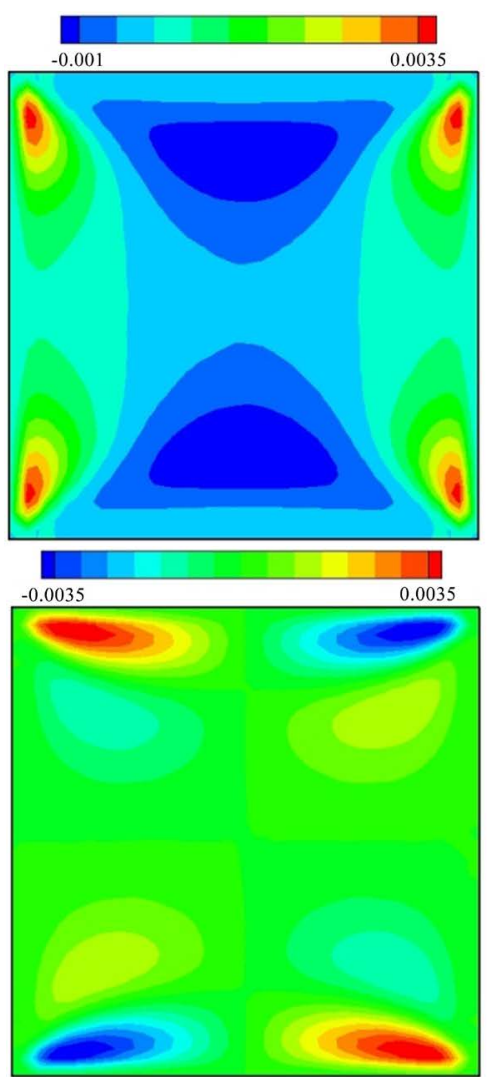

(a) $\mathrm{b}=0.1$
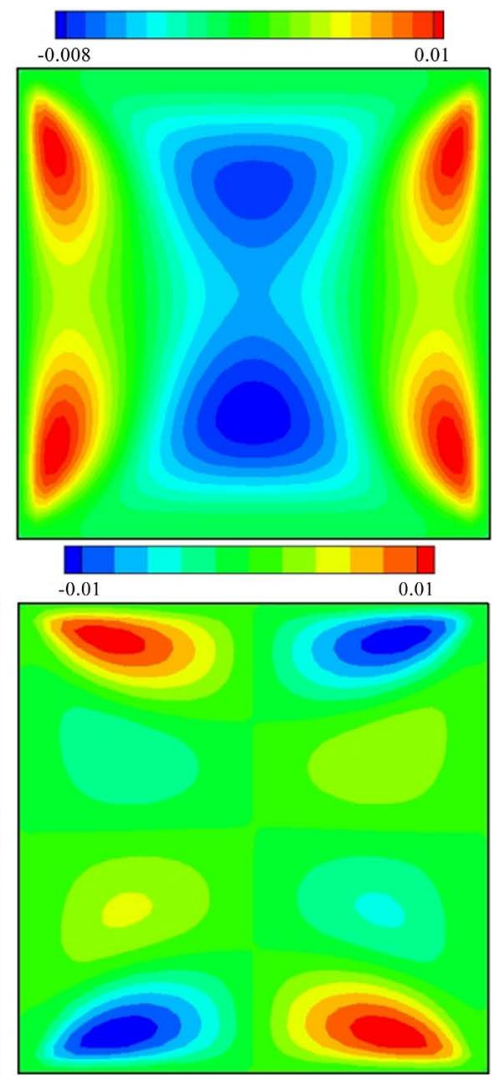

(b) $\mathrm{b}=0.01$
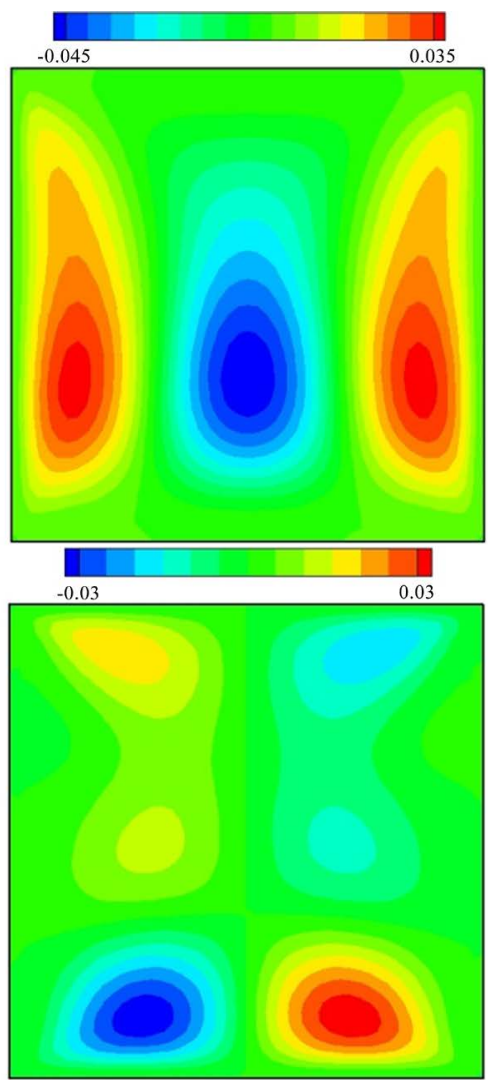

(c) $b=0.001$

Figure 7. The contours of dimensionless vertical velocity (top) and dimensionless horizontal velocity (bottom) at different vertical slip lengths.

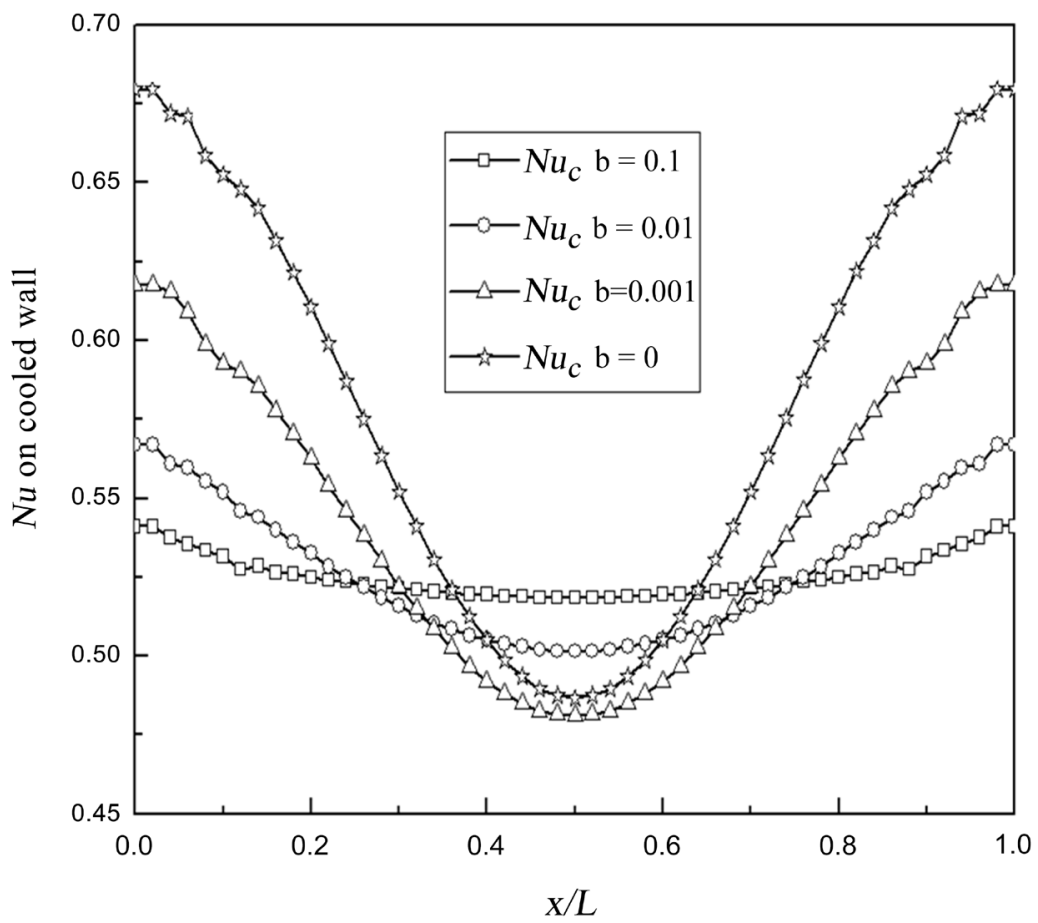

(a) 


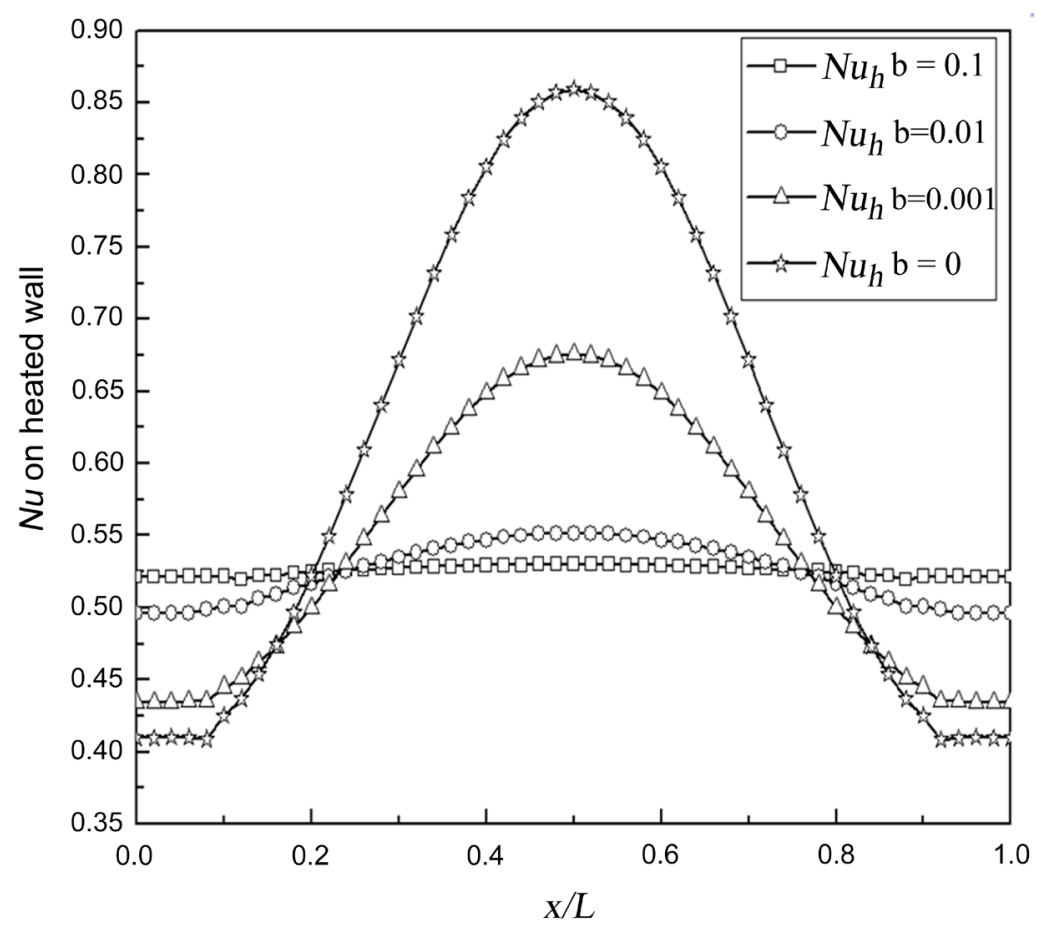

(b)

Figure 8. (a) The local Nusselt number on cooled upper wall; (b) The local Nusselt number on heated bottom wall changing with vertical slip length.

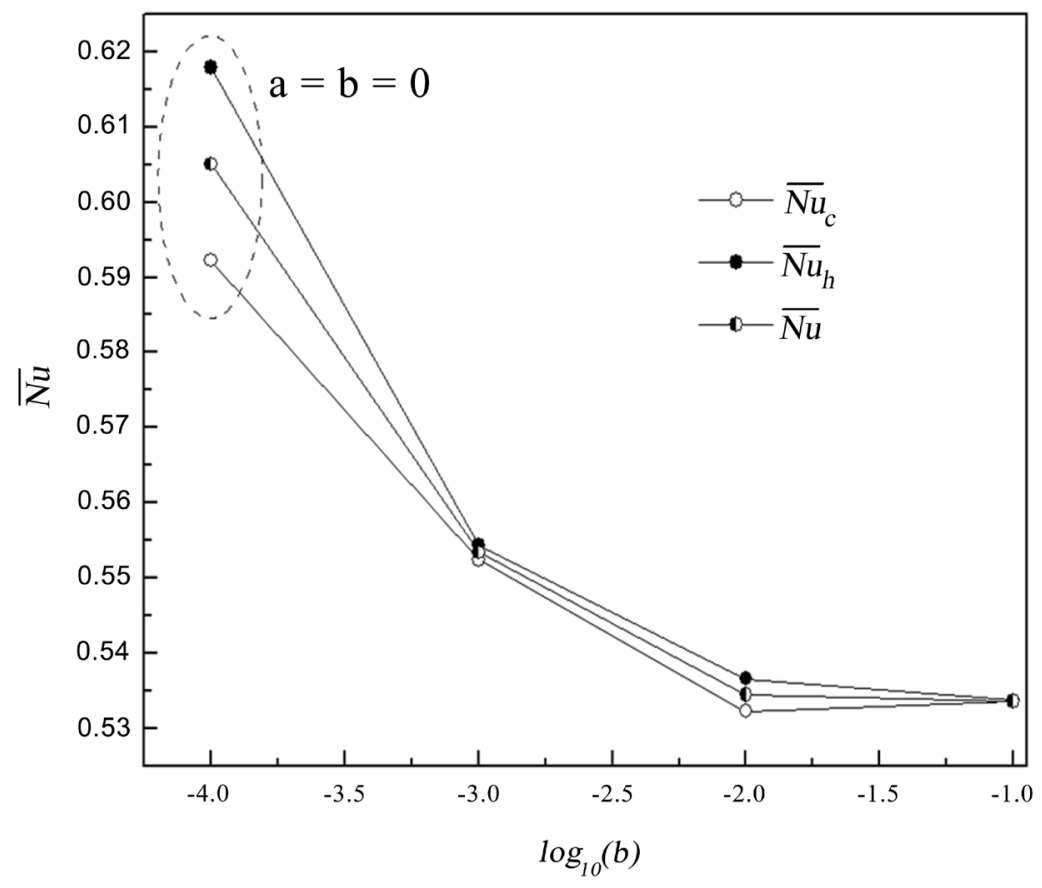

Figure 9. The average Nusselt number changing with vertical slip length.

vection will be restrained, it is need to diminish the vertical slip length to drive the convection. Thus, the critical vertical slip length decreases with the Rayleigh number and the magnetic Rayleigh number. In addition, the effect of the vertical slip length on the convection is obvious when the Rayleigh number and the magnetic Rayleigh number is larger. 


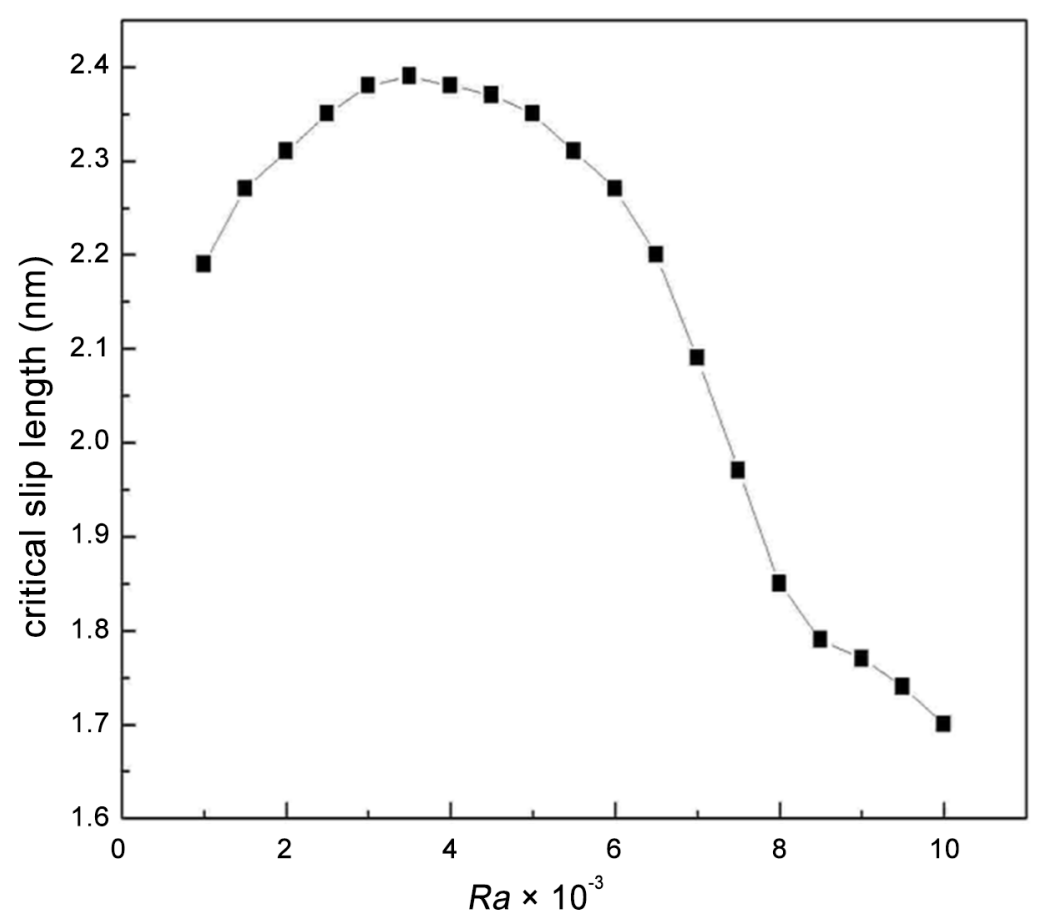

(a)

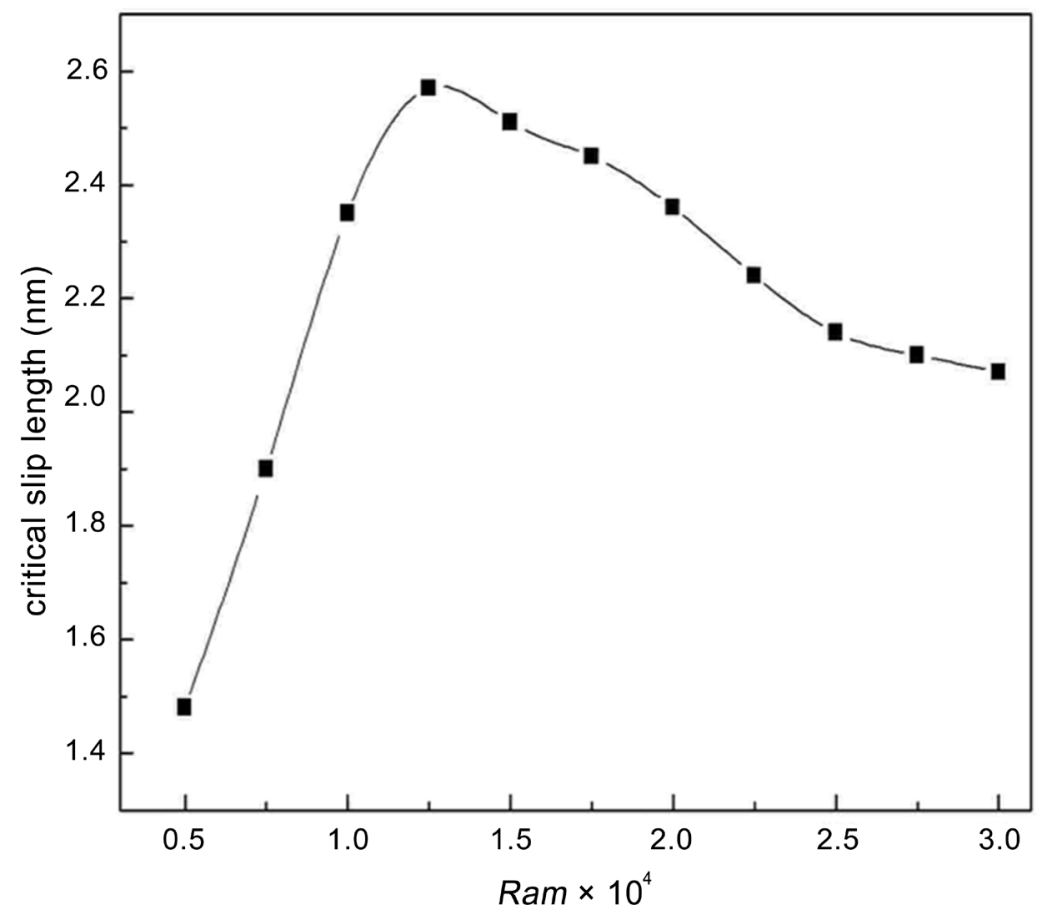

(b)

Figure 10. (a) The critical slip length changing with Rayleigh number; (b) The critical slip length changing with magnetic Rayleigh number.

\subsection{The Condition That the Horizontal and Vertical Slip Length Both Exist}

When the horizontal and vertical slip lengths both exist, we investigate the properties of the convection. In Figure 11, when the slip lengths become larger, the convection will be harder to occur, the vertical and horizontal 
velocity, the vertical and horizontal velocity gradients will decrease. Comparing Figure 11(a) with Figure 2(b) and Figure 6(b), we can find that the vertical and horizontal slip lengths associate with each other, accelerate the flow to be local vortexes, result in more stable convection.

Figure 12 shows that the average Nusselt numbers on the cooled wall, on the heated wall and of the whole cavity decrease with the slip length, the heat transfer efficiency will decrease. Comparing Figure 12 with Figure 5 and Figure 9, when both of the horizontal and vertical slip lengths exist, the variation of the average Nus-

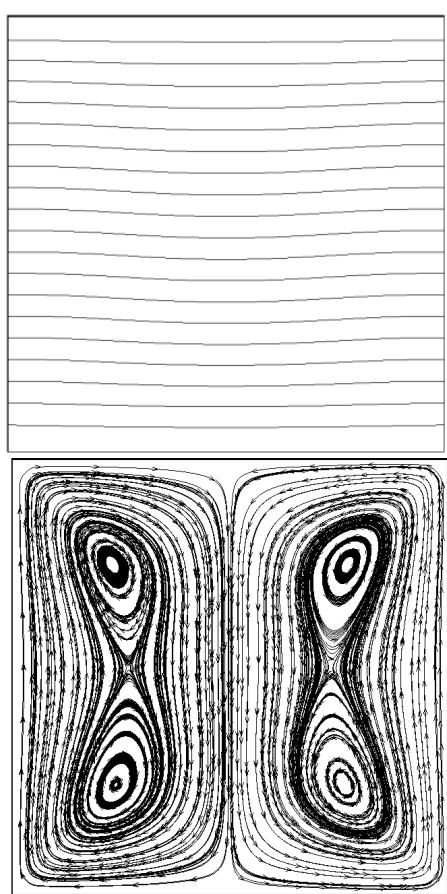

(a)

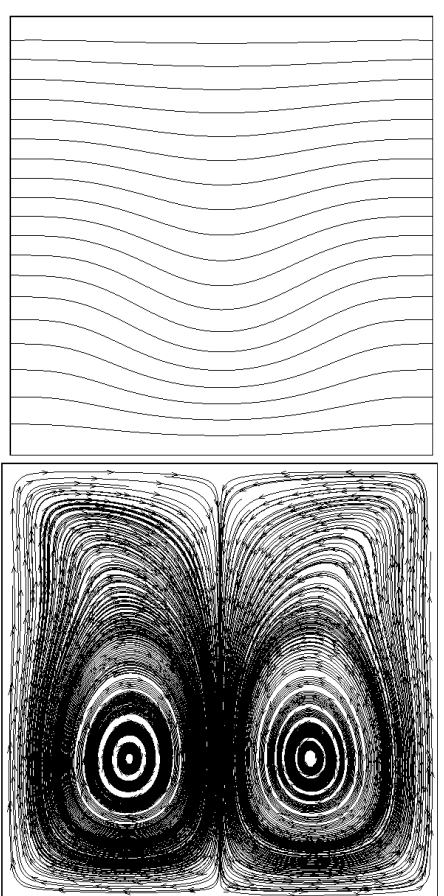

(b)

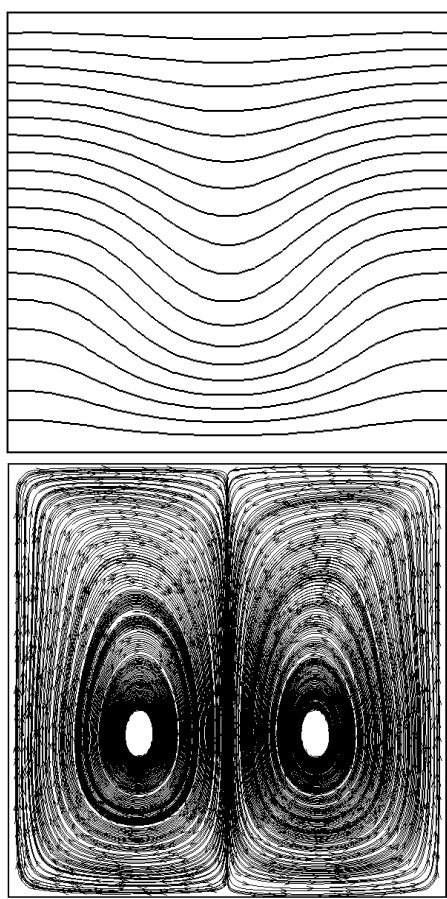

(c)

Figure 11. The streamlines (top) and isotherms (bottom) at different slip lengths (a) $a=b=0.01$; (b) $a=$ $\mathrm{b}=0.001 ;(\mathrm{c}) \mathrm{a}=\mathrm{b}=0$.

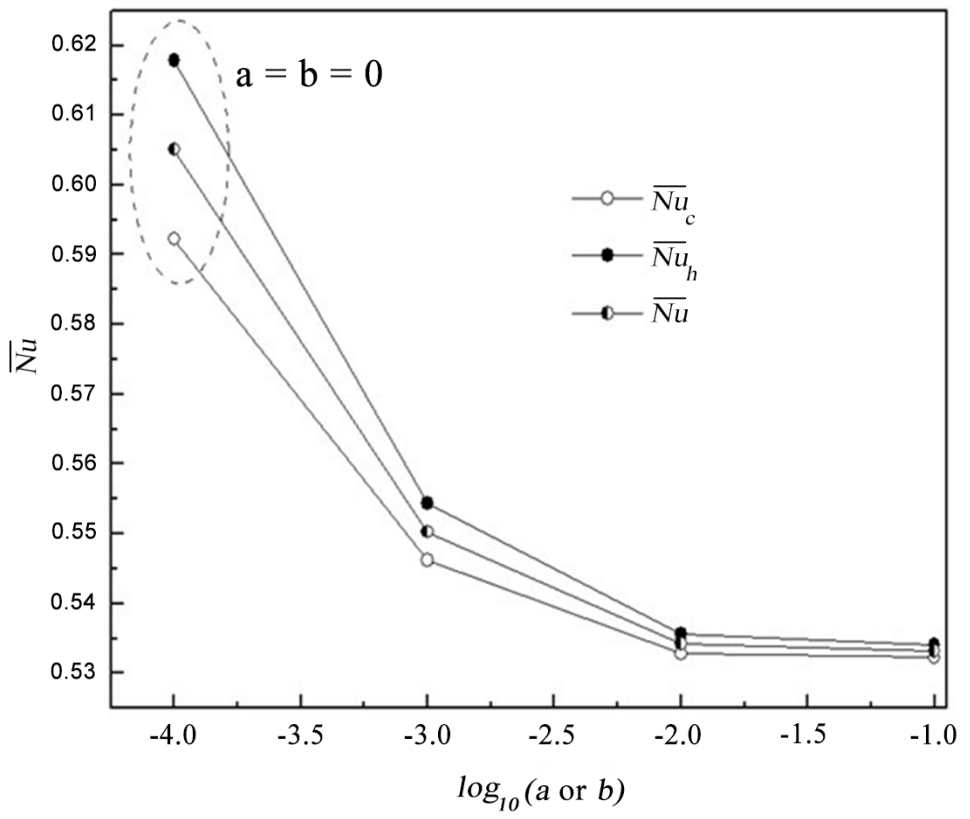

Figure 12. The average Nusselt number changing with slip length. 
selt numbers becomes smooth, it may close to the real situations.

The horizontal and vertical slip lengths are related to the properties of the walls (assuming the properties of the fluid are constant). In micro porous media systems, the slip velocities on the walls are ubiquitous. Therefore, through altering the properties of the walls (the horizontal walls or the vertical walls or both), we can make the convection in the systems more stable or unstable, increase or decrease the heat transfer efficiency. This study may be useful in future applications of the heat accumulation or cooling industry.

\section{Conclusions}

A mathematical model of the convection of temperature-sensitive magnetic fluids in a micro porous cavity is developed. According to Navier's linear slip length model, slip boundary conditions are used on all the walls of the cavity. A Lattice Boltzmann method is used to investigate the effects of the horizontal slip velocity and the vertical slip velocity on the convection. We can conclude that:

1) When the slip length becomes larger, the distribution of temperature will be much gentle; the horizontal and vertical velocities, the horizontal and vertical velocity gradients will be smaller; the convection will be harder to occur.

2) When the horizontal slip length equals to zero, with the increase of the vertical slip length, the convection will be harder to occur, and the flow develops from one vortex to two vortexes along the vertical direction. Therefore, along the vertical direction, when the Rayleigh number and the magnetic Rayleigh number is constant, there exists a critical slip length. The critical vertical slip length increases and then decreases with the Rayleigh number and the magnetic Rayleigh number. In addition, when the Rayleigh number and the magnetic Rayleigh number are larger, the effect of the vertical slip length on the convection becomes more obvious.

3) On the cooled wall, the Nusselt number near side walls decreases with the slip length, but increases in the center of the wall. On the heated wall, the Nusselt number near side walls increases with the slip length, but decreases in the center of the wall.

4) The average Nusselt number of the whole cavity decreases with the slip length, and the heat transfer efficiency will descend. The effect of the vertical slip velocity on the Nusselt number is more obvious than that of the horizontal slip velocity.

\section{Acknowledgements}

The support of the National Science Foundation of China (51276001) is gratefully acknowledged. Supported by Common Development Fund of Beijing is also appreciated.

\section{References}

[1] Nield, D. and Bejan, A. (1998) Convection in Porous Media. Springer, New York.

[2] Miguel, A.F. (2012) Non-Darcy Porous Media Flow in No-Slip and Slip Regimes. Thermal Science, 16, 167-176. http://dx.doi.org/10.2298/TSCI100929001M

[3] Guo, Z. and Zhao, T.S. (2005) Lattice Boltzmann Simulation of Natural Convection with Temperature-Dependent Viscosity in a Porous Cavity. Progress in Computational Fluid Dynamics, 5, 110-117. http://dx.doi.org/10.1504/PCFD.2005.005823

[4] Park, J., Matsubara, M. and Li, X. (2007) Application of Lattice Boltzmann Method to a Micro-Scale Flow Simulation in the Porous Electrode of a PEM Fuel Cell. Journal of Power Sources, 173, 404-414. http://dx.doi.org/10.1016/j.jpowsour.2007.04.021

[5] Wang, M. and Chen, S. (2007) Electroosmosis in Homogeneously Charger Micro- and Nanoscale Random Porous Media. Journal of Colloid and Interface Science, 314, 264-273. http://dx.doi.org/10.1016/j.jcis.2007.05.043

[6] Wang M., Kang Q., Viswanathan, H. and Robinson, B.A. (2010) Modeling of Electro-Osmosis of Dilute Electrolyte Solutions in Silica Microporous Media. Journal of Geophysical Research, 115, B10205. http://dx.doi.org/10.1029/2010JB007460

[7] Tang, G.H., Ye, P.X. and Tao, W.Q. (2010) Pressure-Driven and Electroosmotic Non-Newtonian Flows through Microporous Media via Lattice Boltzmann Method. Journal of Non-Newtonian Fluid Mechanics, 165, 1536-1542.

[8] Craig, V.S.J., Neto, C. and Williams, D.R.M. (2001) Shear-Dependent Boundary Slip in an Aqueous Newtonian Liquid. Physical Review Letters, 87, 054504. http://dx.doi.org/10.1103/PhysRevLett.87.054504

[9] Zhu, Y.X. and Granick, S. (2001) Limits of the Hydrodynamic No-Slip Boundary Condition. Physical Review Letters, 
88, Article ID: 054504.

[10] Cottin-Bizonne C., Cross B., Steinberger, A. and Charlaix, E. (2005) Boundary Slip on Smooth Hydrophobic Surfaces: Intrinsic Effects and Possible Artifacts. Physical Review Letters, 94, Article ID: 056102. http://dx.doi.org/10.1103/PhysRevLett.94.056102

[11] Shirani, E. and Jafari, S. (2007) Application of LBM in Simulation of Flow in Simple Micro-Geometries and Micro Porous Media. African Physical Review, 1, 0002.

[12] Takenaka, S., Suga, K., Kinjo, T. and Hyodo, S. (2009) Flow Simulation in a Sub-Micro Porous Medium by the Lattice Boltzmann and the Molecular Dynamics Methods. The 7th International ASME Conference on Nanochannels, Microchannels and Minichannels, Pohang, 22-24 June 2009, 1-10.

[13] Suga, K., Takenaka, S., Kinjo, T. and Hyodo, S. (2009) LBM and MD Simulations of a Flow in a Nano-Porous Medium. 2nd Asian Symposium on Computational Heat Transfer and Fluid Flow, Jeju, 20-23 October 2009, 112-117.

[14] Thompson, P.A. and Troian, S.M (1997) A General Boundary Condition for Liquid Flow at Solid Surfaces. Nature, 389, 360-362. http://dx.doi.org/10.1038/38686

[15] Sofonea, V. and Sekerka, R.F. (2005) Boundary Conditions for the Upwind Finite Difference Lattice Boltzmann Model: Evidence of Slip Velocity in Micro-Channel Flow. Journal of Computational Physics, 207, 639-659. http://dx.doi.org/10.1016/j.jcp.2005.02.003

[16] Chen, S. and Tian, Z. (2010) Simulation of Thermal Micro-Flow Using Lattice Boltzmann Method with Langmuir Slip Model. International Journal of Heat and Fluid Flow, 31, 227-235. http://dx.doi.org/10.1016/j.ijheatfluidflow.2009.12.006

[17] Yang, F. (2007) Flow Behavior of an Eyring Fluid in a Nanotube: The Effect of the Slip Boundary Condition. Applied Physics Letters, 90, Article ID: 133105. http://dx.doi.org/10.1063/1.2717019

[18] Larrode, F.E., Housiadas, C. and Drossinos, Y. (2000) Slip-Flow Heat Transfer in Circular Tubes. International Journal of Heat and Mass Transfer, 43, 2669-2680. http://dx.doi.org/10.1016/S0017-9310(99)00324-5

[19] Jin, L., Zhang, X. and Niu, X. (2011) Lattice Boltzmann Simulation for Temperature-Sensitive Magnetic Fluids in a Porous Square Cavity. Journal of Magnetism and Magnetic Materials, 324, 44-51. http://dx.doi.org/10.1016/j.jmmm.2011.07.033

[20] Jin, L. and Zhang, X. (2013) Analysis of Temperature-Sensitive Magnetic Fluids in a Porous Square Cavity Depending on Different Porosity and Darcy Number. Applied Thermal Engineering, 50, 1-11. http://dx.doi.org/10.1016/j.applthermaleng.2012.05.016 